\title{
RANK/RANK-L/OPG in Patients with Bone Metastases Treated with Anticancer Agents and Zoledronic Acid: A Prospective Study
}

Laura Mercatali ${ }^{1,2}$, Marianna Ricci ${ }^{1}$, Emanuela Scarpi ${ }^{3}$, Patrizia Serra ${ }^{3}$, Francesca Fabbri ${ }^{3}$, Rossana Ricci ${ }^{1}$, Chiara Liverani ${ }^{1,2}$, Michele Zanoni ${ }^{2}$, Wainer Zoli ${ }^{2}$, Roberta Maltoni ${ }^{4}$, Erica Gunelli ${ }^{1}$, Dino Amadori ${ }^{1}$ and Toni Ibrahim ${ }^{1, *}$

1 Osteoncology and Rare Tumors Center, IRCCS Istituto Scientifico Romagnolo per lo Studio e la Cura dei Tumori (IRST), via P. Maroncelli 40, 47014 Meldola (FC), Italy;

E-Mails: 1.mercatali@irst.emr.it (L.M.); marianna.ricci@irst.emr.it (M.R.); rossana.ricci@ausl.fo.it (R.R.); c.liverani@irst.emr.it (C.L.); ee219@libero.it (E.G.); d.amadori@irst.emr.it (D.A.)

2 Biosciences Laboratory, IRCCS Istituto Scientifico Romagnolo per lo Studio e la Cura dei Tumori (IRST), via P. Maroncelli 40, 47014 Meldola (FC), Italy; E-Mails: m.zanoni@irst.emr.it (M.Z.); w.zoli@irst.emr.it (W.Z.)

3 Unit of Biostatistics and Clinical Trials, IRCCS Istituto Scientifico Romagnolo per lo Studio e la Cura dei Tumori (IRST), via P. Maroncelli 40, 47014 Meldola (FC), Italy;

E-Mail: e.scarpi@irst.emr.it (E.S.); p.serra@irst.emr.it (P.S.); francesca.fabbri@irst.emr.it (F.F.)

4 Department of Medical Oncology, IRCCS Istituto Scientifico Romagnolo per lo Studio e la Cura dei Tumori (IRST), via P. Maroncelli 40, 47014 Meldola (FC), Italy; E-Mail: r.maltoni@irst.emr.it

* Author to whom correspondence should be addressed; E-Mail: t.ibrahim@irst.emr.it; Tel.: +39-543-739-239; Fax: +39-543-739-221.

Received: 15 April 2013; in revised form: 9 May 2013 / Accepted: 13 May 2013 /

Published: 23 May 2013

Abstract: Patients with solid cancer frequently develop bone metastases (BM). Zoledronic acid (Zometa $\left.{ }^{\circledR}, \mathrm{ZA}\right)$, routinely used to treat patients with BM, acts on osteoclasts and also has antitumor properties. We aimed to assess the effect of ZA over time in novel bone turnover markers (RANK/receptor activator of nuclear factor-k B ligand (RANK-L)/ Osteoprotegerin (OPG)) and to correlate these with serum N-terminal telopeptide (NTX). The study prospectively evaluated levels of RANK, RANK-L and OPG transcripts by real-time PCR and NTX expression by ELISA in the peripheral blood of 49 consecutive patients with advanced breast, lung or prostate cancer. All patients received the standard 
ZA schedule and were monitored for 12 months. Median baseline values of RANK, RANK-L and OPG were 78.28 (range 7.34-620.64), 319.06 (21.42-1884.41) and 1.52 (0.10-58.02), respectively. At 12 months, the median RANK-L value had decreased by $22 \%$ with respect to the baseline, whereas median OPG levels had increased by about $96 \%$. Consequently, the RANK-L/OPG ratio decreased by $56 \%$ from the baseline. Median serum NTX levels decreased over the 12-month period, reaching statistical significance $(p<0.0001)$. Our results would seem to indicate that ZA modulates RANK, RANK-L and OPG expression, thus decreasing osteoclast activity.

Keywords: RANK; RANK-L; OPG; NTX; bone metastases

\section{Introduction}

Bone metastases are common in many solid tumors, in particular breast, prostate and lung cancer. In the United States, about two-thirds of patients who die from cancer each year have metastatic bone disease $[1,2]$. Approximately $20 \%-25 \%$ of patients with cancer develop clinically evident bone metastases, and a further $50 \%$ of these have lesions identified at autopsy [3,4]. Metastatic bone disease disrupts normal bone homeostasis, a dynamic process involving osteoclast-mediated osteolysis and osteoblast-mediated osteogenesis. Increasingly unbalanced bone metabolism leads to a loss of bone integrity, which may result in skeletal-related events (SREs), such as bone pain, pathological fractures, spinal cord compression or hypercalcemia. The frequency of SREs in these patients depends mainly on the site of metastasis, type of lesion (lytic or blastic) and previous treatment received [5].

Bisphosphonates play a key role in the treatment of bone metastases [6], positively influencing the natural history of the disease, improving quality of life and decreasing pain and the frequency of skeletal-related events. The most widely used bisphosphonate is zoledronic acid (ZA) [6-9]. Several circulating markers have been studied for their potential to predict response to ZA in patients with bone metastases, without, however, encouraging results. Urinary $n$-telopeptide (NTX) is the marker most frequently analyzed in studies of large case series [10-15], and normalization of urinary NTX values after three months' treatment with ZA has been associated, in retrospective studies, with reduced risks of both skeletal complications and death [16].

The molecules involved in the RANK/RANK-L/OPG axis, which governs osteoclastogenesis and bone resorption, are also candidate markers $[17,18]$. The receptor activator of nuclear factor-k B ligand (RANK-L) binds and activates its receptor, RANK, on the surface of osteoclasts, stimulating osteoclast differentiation and maturation, inhibiting osteoclast apoptosis and increasing bone resorption. Osteoprotegerin (OPG), a member of the TNF receptor family, is secreted by various cell types, including osteogenic cells, and acts as a decoy receptor of RANK-L, thereby inhibiting osteoclastogenesis. It has been hypothesized that the RANK/RANK-L/OPG pathway may be modulated by ZA, because of its involvement in the modulation of bone resorption.

Circulating bone turnover markers of resorption and formation have been evaluated for their ability to act as indicators of activity of bisphosphonates in metastatic bone disease. 
The aim of this prospective study was to evaluate the effect of ZA over time on novel circulating bone turnover markers (RANK/RANK-L/OPG) and to correlate these with serum NTX.

\section{Results}

Patient characteristics are listed in Table 1. Biological and pathological data on breast cancer patients are reported on Table 2, and clinical data on bone lesions and other sites of distant metastases are shown in Table 3.

Table 1. Patient characteristics: overall case series.

\begin{tabular}{ccccc}
\hline & \multicolumn{4}{c}{ Cancer patients } \\
\hline Features & Total & $\mathrm{BC} *$ & $\mathrm{PCa}^{\S}$ & Lung \\
& No. $(\%)$ & $\mathrm{No.}(\%)$ & $\mathrm{No.}(\%)$ & No. $(\%)$ \\
Overall & 49 & 36 & 7 & 6 \\
Median age, years (range) & $62(34-86)$ & $59(34-86)$ & $70(51-83)$ & $60(47-68)$ \\
Sex: males & $12(24.5)$ & 0 & $7(100)$ & $5(83.3)$ \\
females & $37(75.5)$ & $36(100)$ & 0 & $1(16.7)$ \\
Performance status & & & & \\
0 & $40(81.6)$ & $30(83.3)$ & $6(85.7)$ & $4(66.7)$ \\
1 & $8(16.3)$ & $6(16.7)$ & 0 & $2(33.3)$ \\
2 & $1(2.1)$ & 0 & $1(14.3)$ & 0 \\
\hline
\end{tabular}

Note: ${ }^{*} \mathrm{BC}$, breast cancer; ${ }^{\S} \mathrm{PCa}$, prostate cancer.

Table 2. Bio-pathological features of BC primary tumors.

\begin{tabular}{cc}
\hline Features & No. $(\%)$ \\
\hline Histology & $21(58.3)$ \\
Ductal & $8(22.3)$ \\
Lobular & $7(19.4)$ \\
Adenocarcinoma & \\
Grading & $2(5.5)$ \\
1 & $14(38.9)$ \\
2 & $20(55.6)$ \\
3 & \\
ER $*$ & $3(8.3)$ \\
$<10 \%$ & $33(91.7)$ \\
$\geq 10 \%$ & $12(33.3)$ \\
PgR & \\
$<10 \%$ & $24(66.7)$ \\
$\geq 10 \%$ & \\
Ki67 & $12(33.3)$ \\
Low & $24(66.7)$ \\
High & \\
HER2 & $23(63.9)$ \\
Not amplified & $13(36.1)$ \\
Amplified &
\end{tabular}


Table 2. Cont.

\begin{tabular}{cc}
\hline Features & No. $(\%)$ \\
\hline Menopausal status & $6(16.7)$ \\
Premenopausal & $2(5.5)$ \\
Perimenopausal & $28(77.8)$ \\
Postmenopausal & \\
Therapies & $3(9.4)$ \\
Neoadjuvant & $28(82.4)$ \\
Adjuvant & $24(75.0)$ \\
Chemotherapy & $26(81.2)$ \\
Hormone & $3(9.7)$ \\
Biological & \\
Lines of therapy & $16(44.5)$ \\
1 & $13(36.1)$ \\
2 & $7(19.4)$ \\
3
\end{tabular}

Note: ${ }^{*}$ ER, estrogen receptor; ${ }^{\S} \mathrm{PgR}$, progesterone receptor.

Table 3. Metastasis features of cancer patients. BM, bone metastases. SREs, skeletal-related events.

\begin{tabular}{ccccc}
\hline & \multicolumn{4}{c}{ Cancer Patients } \\
\cline { 2 - 5 } & Total (\%) & BC (\%) & PCa (\%) & Lung (\%) \\
\hline Visceral metastases & & & & \\
Presence & $25(51.0)$ & $15(41.7)$ & $5(71.4)$ & $5(83.3)$ \\
Absence & $24(49.0)$ & $21(58.3)$ & $2(28.6)$ & $1(16.7)$ \\
\hline Bone lesion type & & & & \\
Osteolytic & $24(55.8)$ & $20(58.8)$ & $1(25.0)$ & $3(60.0)$ \\
Osteoblastic & $13(30.2)$ & $10(29.4)$ & $3(75.0)$ & 0 \\
Mixed & $6(14.0)$ & $4(11.8)$ & 0 & $2(40.0)$ \\
Missing & 6 & 2 & 3 & 1 \\
\hline No. of BM sites & $5(10.2)$ & $4(11.1)$ & $1(14.3)$ & 0 \\
1 & $34(69.4)$ & $25(69.4)$ & $4(57.1)$ & $5(83.3)$ \\
$2-6$ & $10(20.4)$ & $7(19.4)$ & $2(28.6)$ & $1(16.7)$ \\
$>6$ & 7 & $6(85.7)$ & $1(14.3)$ & 0 \\
\hline No. of SREs & \multicolumn{5}{c}{} &
\end{tabular}

\subsection{RANK/RANK-L/OPG Pathways}

Considering the entire case series, median baseline RANK, RANK-L and OPG values were 78.28 (range 7.34-620.64), 319.06 (21.42-1884.41) and 1.52 (0.10-58.02), respectively. At 9-12 months, median RANK-L values had decreased by $22 \%$ with respect to the baseline, while median OPG levels had increased by about $96 \%$. Consequently, the RANK-L/OPG ratio decreased by $56 \%$ from the baseline. These differences, however, did not reach statistical significance. Median RANK values had risen by $36 \%$ at 6-8 months, but showed a return towards baseline values at 9-12 months $(p=0.013)$ (Figure 1). OPG, RANK and RANK-L/OPG ratio levels were significantly related. 
Figure 1. Marker variations over time.

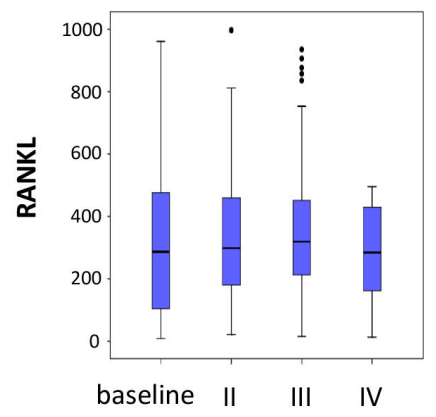

Time Points
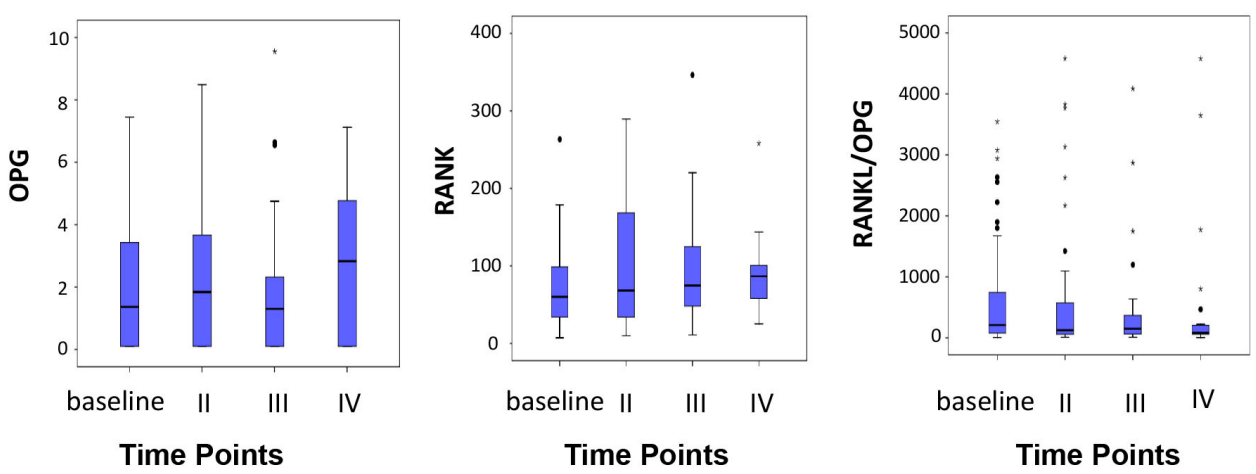

Time Points

\subsection{NTX}

The median baseline NTX value was $15.65 \mathrm{~nm}$ bone collagen equivalent BCE (range 2.85-45.00). Median values were $10.80 \mathrm{~nm}$ BCE (4.62-34.91) at 3-4 months, $9.99 \mathrm{~nm} \mathrm{BCE} \mathrm{(3.64-21.2)} \mathrm{at} \mathrm{6-8}$ months and $10.91 \mathrm{~nm} \mathrm{BCE} \mathrm{(5.53-45.00)} \mathrm{at} \mathrm{9-12} \mathrm{months.} \mathrm{Median} \mathrm{serum} \mathrm{NTX} \mathrm{levels} \mathrm{at} \mathrm{3-4} \mathrm{and} \mathrm{at} \mathrm{6-8}$ months were $35 \%$ and $39 \%$ lower than those of baseline, respectively, reaching statistical significance $(p<0.0001)$. The median NTX value at 9-12 months showed a 26\% decrease with respect to the baseline (Figure 2).

Figure 2. N-terminal telopeptide (NTX) variation over time.

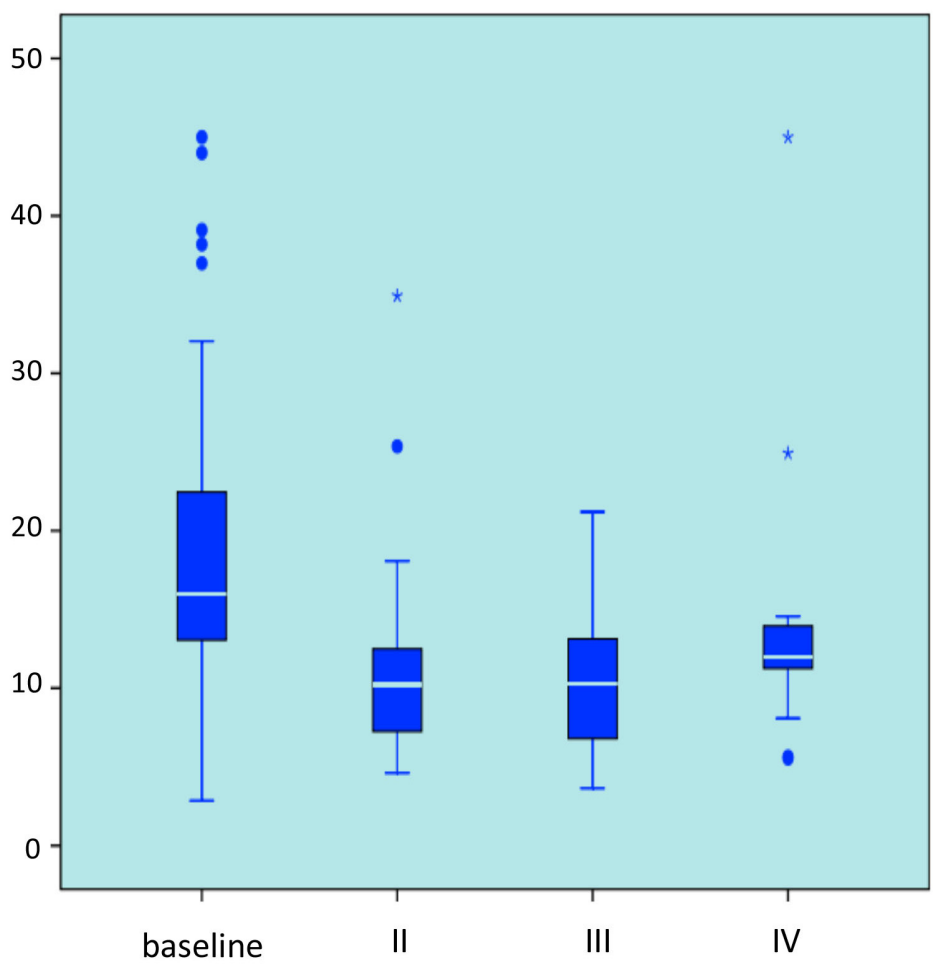

Time Points 


\subsection{Breast Cancer Patients}

CEA and CA15-3

The median baseline CEA value of $7.32 \mathrm{ng} / \mathrm{mL}$ (range $25.12-1264.09$ ) had decreased to 4.10 (1.00-1122.10) at 9-12 months (not statistically significant). CA15-3 showed a median baseline value of $40.00 \mathrm{ng} / \mathrm{mL}$ (range $1.50-809.00$ ), increasing to 48.60 (6.20-1633.00) at 9-12 months (not statistically significant). No correlation was found between CEA and CA15-3 and the other markers evaluated.

\section{Discussion}

In a previous case control study, we observed that median OPG levels of bone metastases were significantly lower in BM patients compared to disease-free individuals or healthy donors [19]. Furthermore, no significant differences were seen in RANK-L and OPG levels as a function of age and menopausal status. Taking into consideration the role played by zoledronic acid in the inhibition of bone turnover, we decided to evaluate the RANK/RANK-L/OPG pathway to understand whether ZA also exerts its effect on bone resorption through this pathway. To our knowledge, this is one of the few prospective studies to assess circulating marker changes in response to ZA in patients with bone metastases from solid tumors. We evaluated about fifty patients, $73 \%$ of whom had breast cancer as the primary tumor. Results were interesting because, after 12 months of treatment (12 ZA infusions), we observed a decrease in RANK-L in the overall case series, accompanied by an increase in OPG, with a consequent decrease in the RANK-L/OPG ratio. This last event has important biological implications, suggesting that ZA over time may influence the expression pattern of molecules involved in pre-osteoclast activation. The RANK trend over time was less linear than that of RANK-L or OPG, showing an increase at 6-8 months and a return towards baseline values at 9-12 months. Such heterogeneity could be explained by the fact that RANK-L and OPG are produced mainly by bone and immune system cells, whereas RANK expression may depend on cancer cell expression [20,21]. We only obtained statistically significant results for NTX [22-26]. In a similarly sized case series, Mountzios and coworkers observed a decrease in the RANK-L/OPG ratio, albeit not statistically significant, following a reduction in only RANK-L values. It must be pointed out here that we measured mRNA extracted from whole blood, whereas Mountzios evaluated protein concentrations from serum samples, stating that results should be interpreted with caution, because RANK-L measures were invalidated by the limited stability of the serum isoform.

Our evaluation of NTX, widely used to monitor metastatic bone disease, confirmed previous observations of a significant decrease in the marker after ZA treatment. Interestingly, this decrease was already present at the first follow-up evaluation. NTX was not related to other markers and showed different behavior when analyzed by subgroup. The RANK-L/OPG ratio was found to be a prognostic factor for survival in multiple myeloma [27].

CEA and CA15-3, markers used in routine clinical practice for breast cancer patients, showed no correlation with other markers. Zhao and coworkers reported a normalization of CEA and CA15-3 values in patients treated with weekly ZA rather than the standard monthly schedule [22]. We did not monitor the role of CEA and PSA in lung and prostate cancer, respectively, because of the small 
number of patients in our study. Our results would seem to indicate that markers of the RANK/RANK-L/OPG pathway could be better at identifying neoplastic bone involvement than conventional tumor markers.

\section{Materials and Methods}

\subsection{Study Design}

This prospective study was carried out at IRCCS Istituto Scientifico Romagnolo per lo Studio e la Cura dei Tumori in Meldola, Italy. Our primary objective was to evaluate the trend of circulating markers over time. Chosen markers were OPG, RANK-L and RANK transcripts and serum NTX protein levels. We also aimed to correlate results with those obtained using conventional tumor markers, carcinoembryonic antigen (CEA) and CA15-3. Furthermore, all markers were correlated with biological parameters of the primary tumor. The protocol was reviewed and approved by the local ethics committee and performed according to Good Clinical Practice and the Helsinki declaration. The patients gave their written informed consent to take part in the study.

\subsection{Case Series}

Peripheral venous blood (PB) samples were obtained from 49 consecutive patients with bone metastases from solid tumors consecutively enrolled from March 2007 to December 2009. Thirty-six patients had breast cancer, 7 prostate cancer and 6 lung cancer. Eligibility criteria were as follows: males/females age $\geq 18$ years; histological confirmation of solid cancer; radiological confirmation of bone metastases; performance status $\leq 2$; life expectancy $>6$ months; normal renal and hepatic function with total bilirubin $<2 \mathrm{mg} / \mathrm{dL}$; and serum creatinine $<2 \mathrm{mg} / \mathrm{dL}$. Exclusion criteria comprised: history of postmenopausal osteoporosis; previous treatment with bisphosphonates, presence of osteonecrosis of the jaw and dental conditions requiring oral cavity surgery. None of the patients had active cardiac disease.

\subsection{Treatment, Blood Collection, Follow Up}

Patients received a 15 min intravenous infusion of ZA $4 \mathrm{mg}$ (Zometa, Novartis, East Hanover, NJ, USA) in $100 \mathrm{~mL}$ of $0.9 \%$ saline every 28 days. Follow up consisted of blood tests and instrumental radiological exams every 3-4 months after the diagnosis of bone metastases for about one year.

The first blood sample was collected at the moment of the bone metastasis diagnosis, before any specific treatment.

\subsection{Biological Samples}

PB samples were collected at each re-evaluation, left to coagulate for $30 \mathrm{~min}$ at room temperature and centrifuged at $2,000 \times \mathrm{g}$ for $15 \mathrm{~min}$, after which serum was stored at $-80{ }^{\circ} \mathrm{C}$ until assays were performed. Pax-gene tubes were left at least two hours at room temperature, stored overnight at $-20{ }^{\circ} \mathrm{C}$ and then at $-80^{\circ} \mathrm{C}$ until RNA extraction was performed. 


\section{5. $R A N K / R A N K-L / O P G$}

Blood RNA was extracted by PAX-Gene blood RNA kit (PreAnalytix-Qiagen, Hilden, Germany) according to the manufacturer's instructions. RNA was treated with DNAse I (Qiagen, Hilden, Germany), and $500 \mathrm{ng}$ of RNA were reverse-transcribed using the iScript cDNA Synthesis Kit (BioRad, Hercules, CA, USA). Real-time PCR was performed using the MyiQ Single Color Real-Time PCR Detection System (BioRad, Hercules, CA, USA) and SYBR Green I dye chemistry. The stably expressed endogenous, $\beta$-actin and HPRT genes, were amplified and used as reference genes. Primers were designed by Beacon Designer Software (Premier Biosoft International, Palo Alto, CA, USA). Primer sequences and the real-time PCR thermic profile have been reported previously [28]. The type of analysis is a relative expression of the transcript with respect to the mRNA of a healthy donor: for this reason, the expression of RANK/RANK-L/OPG do not need a unit.

\subsection{NTX Immunoassay}

NTX levels were measured by a competitive-inhibition enzyme-linked immunosorbent assay (ELISA/EIA) (Osteomark, Princeton, NJ, USA). The assays were performed following the manufacturer's instructions. All samples were tested in duplicate for both markers. For both analyses, all samples from the same individuals were analyzed on the same experimental plate. Immunoassays were performed according to the manufacturers' instructions.

With regard to intra-assay reproducibility, all determinations were performed in duplicate and repeated when the coefficient of variation (CV) exceeded 10\%. An internal control was added in all assays to assess inter-assay reproducibility. Inter-assay CV was always less than $15 \%$.

\subsection{Carcinoembryonic Antigen and CA15-3}

CEA and CA 15-3 assays were routinely performed in the Clinical Pathology Laboratory of Morgagni-Pierantoni Hospital in Forlì using AxSYM Chemiluminescent Microparticle Immunoassay (CMIA) and Architect Microparticle Enzyme Immunoassay (MEIA; Abbott Laboratories, Abbott Park, III, USA), respectively. The markers were only used to evaluate breast cancer patients.

\subsection{Statistical Analysis}

The median value of each marker, considered as a continuous variable, was calculated at the time of the diagnosis of bone involvement ("baseline" measurement) and every 3-4 months thereafter.

A comparison of marker values over time (observations repeated in the same subjects) was made using nonparametric ranking statistics (Friedman test). Another nonparametric ranking statistic method, the median test, was used to analyze the relationship between serum levels of each marker and patient characteristics. Spearman's rank correlation test was used to investigate the relation between baseline values of each marker. Statistical analyses were carried out with SAS Statistical software (version 9.1, SAS Institute, Cary, NC, USA). 


\section{Conclusions}

In conclusion, the present prospective study highlights a clear trend towards a decrease in the RANK-L/OPG ratio and NTX levels after 12 months of treatment with zoledronic acid. Our findings concerning the decrease in the RANK-L/OPG ratio raises the interesting hypothesis that this drug may also influence osteoclast behavior during differentiation, perhaps via an indirect mechanism that leads to a block in the vicious cycle of bone metastases. Further investigations into this area are warranted.

\section{Acknowledgements}

The authors thank Ursula Elbling for editing the manuscript. LM and TI conceived and designed the study and drafted the manuscript. FF, CL, MZ and PS were involved in data acquisition. LM performed the biological determinations. RR was responsible for blood collection and acquisition of clinical data. ES performed the statistical analysis. EG and RM were responsible for acquisition of clinical data. WZ and DA critically revised the manuscript for intellectual content. All authors approved the final version of the manuscript.

\section{Conflicts of Interest}

The authors declare that they have no conflict of interests.

\section{References}

1. Mundy, G.R. Metastasis to bone: Causes, consequences and therapeutic opportunities. Nat. Rev. Cancer 2002, 2, 584-593.

2. Ibrahim, T.; Barbanti, F.; Giorgio-Marrano, G.; Mercatali, L.; Ronconi, S.; Vicini, C.; Amadori, D. Osteonecrosis of the jaw in patients with bone metastases treated with bisphosphonates: A retrospective study. Oncologist 2008, 3, 330-336.

3. Jemal, A.; Siegel, R.; Ward, E.; Murray, T.; Xu, J.; Thun, M.J. Cancer statistics 2007. CA Cancer J. Clin. 2007, 57, 43-66.

4. Coleman, R.E. Clinical features of metastatic bone disease and risk of skeletal morbidity. Clin. Cancer. Res. 2006, 12, 6243s-6249s.

5. Amadori, A.; Cascinu, S.; Conte, P.; Ibrahim, T. Osteoncology Textbook; Poletto Editore: Milan, Italy, 2010.

6. Lipton, A. Zoledronic acid: Multiplicity of use across the cancer continuum. Expert Rev. Anticancer Ther. 2011, 7, 999-1012.

7. Lipton, A.; Theriault, R.L.; Hortobagyi, G.N.; Simeone, J.; Knight R.D.; Mellars, K.; Reitsma, D.J.; Heffernan, M.; Seaman, J. Pamidronate prevents skeletal complications and its effective palliative treatment in women with breast carcinoma and osteolytic bone metastases: Long term follow up of two randomized, placebo-controlled trials. Cancer 2000, 88, 1082-1090.

8. Saad, F. Clinical benefit of zoledronic acid for the prevention of skeletal complications in advanced prostate cancer. Clin. Prostate Cancer 2005, 4, 31-37. 
9. Rosen, L.S.; Gordon, D.; Tchekmedyian, S.; Yanagihara, R., Hirsh, V.; Krzakowski, M.; Pawlicki, M.; de Souza, P.; Zheng, M.; Urbanowitz, G.; et al. Zoledronic acid versus placebo in the treatment of skeletal metastases in patients with lung cancer and other solid tumors: A phase III, double-blind, randomized trial-The zoledronic acid lung cancer and other solid tumors study group. J. Clin. Oncol. 2003, 21, 3150-3157.

10. Coleman, R.; Brown, J.; Terpos, E.; Lipton, A.; Smith, M.R.; Cook, R.; Major, P. Bone markers and their prognostic value in metastatic bone disease: Clinical evidence and future directions. Cancer Treat. Rev. 2008, 34, 629-639.

11. Coleman, R.E. The role of bone markers in metastatic bone disease. Cancer Treat. Rev. 2006, 32 , $\mathrm{S} 1-\mathrm{S} 2$.

12. Garnero, P.; Buchs, N.; Zekri, J.; Rizzoli, R.; Coleman, R.E.; Delmas, P.D. Markers of bone turnover for the management of patients with bone metastases from prostate cancer. Br. J. Cancer 2000, 82, 858-864.

13. Ulrich, U.; Rhiem, K.; Schmolling, J.; Flaskamp, C.; Paffenholz, I.; Sälzer, H.; Bauknecht, T.; Schlebusch, H. Cross-linked type I collagen C- and N-telopeptides in women with bone metastases from breast cancer. Arch. Gynecol. Obstet. 2001, 264, 186-190.

14. Jung, K.; Lein, M.; Stephan, C.; von hösslin, K.; Semjonow, A.; Sinha, P.; Loening, S.A.; Schnorr, D. Comparison of 10 serum bone turnover markers in prostate carcinoma patients with bone metastatic spread: Diagnostic and prognostic implications. Int. J. Cancer 2004, 111, 783-791.

15. Kamiya, N.; Suzuki, H.; Yano, M.; Endo, T.; Takano, M.; Komaru, A.; Kawamura, K.; Sekita, N.; Imamoto, T.; Ichikawa, T. Implications of serum bone turnover markers in prostate cancer patients with bone metastasis. Urology 2010, 75, 1446-1451.

16. Hatoum, H.T.; Lin, S.J.; Smith, M.R.; Barghout, V.; Lipton, A. Zoledronic acid and skeletal complications in patients with solid tumors and bone metastases: Analysis of a national medical claims database. Cancer 2008, 113, 1438-1445.

17. Hofbauer, L.C.; Neubauer, A.; Heufelder, A.E. Receptor activator of nuclear factor-kappaB ligand and osteoprotegerin: Potential implications for the pathogenesis and treatment of malignant bone diseases. Cancer 2001, 92, 460-470.

18. Blair, J.M.; Zhou, H.; Seibel, M.J.; Dunstan, C.R. Mechanisms of disease: Roles of OPG, RANK-L and RANK in the pathophysiology of skeletal metastasis. Nat. Clin. Pract. Oncol. 2006, 3, 41-49.

19. Nicolini, A.; Tartarelli, G.; Carpi, A.; Metelli, M.R.; Ferrari, P.; Anselmi, L.; Conte, M.; Berti, P.; Miccoli, P. Intensive post-operative follow-up of breast cancer patients with tumour markers: CEA, TPA or CA15.3 vs. MCA and MCA-CA15.3 vs. CEA-TPA-CA15.3 panel in the early detection of distant metastases. BMC Cancer 2006, 6, 269.

20. Mountzios, G.; Dimopoulos, M.A.; Bamias, A.; Papadopoulos, G.; Kastritis, E.; Syrigos, K.; Pavlakis, G.; Terpos, E. Abnormal bone remodeling process is due to an imbalance in the receptor activator of nuclear factor-kappaB ligand (RANK-L)/osteoprotegerin (OPG) axis in patients with solid tumors metastatic to the skeleton. Acta. Oncol. 2007, 46, 221-229.

21. Mountzios, G.; Terpos, E.; Syrigos, K.; Papadimitriou, C.; Papadopoulos, G.; Bamias, A.; Mavrikakis, M.; Dimopoulos, M.A. Markers of bone remodeling and skeletal morbidity in patients with solid tumors metastatic to the skeleton receiving the biphosphonate zoledronic acid. Transl. Res. 2010, 155, 247-255. 
22. Zhao, X.; Xu, X.; Guo, L.; Ragaz, J.; Guo, H.; Wu, J.; Shao, Z.; Zhu, J.; Guo, X.; Chen, J.; et al. Biomarker alterations with metronomic use of low-dose zoledronic acid for breast cancer patients with bone metastases and potential clinical significance. Breast Cancer Res. Treat. 2010, 124, 733-743.

23. Lipton, A.; Cook, R.J.; Major, P.; Smith, M.R.; Coleman, R.E. Zoledronic acid and survival in breast cancer patients with bone metastases and elevated markers of osteoclast activity. Oncologist 2007, 12, 1035-1043.

24. Coleman, R.E.; Major, P.; Lipton, A.; Brown, J.E.; Lee, K.A.; Smith, M.; Saad, F.; Zheng, M.; Hei, Y.J.; Seaman, J.; et al. Predictive value of bone resorption and formation markers in cancer patients with bone metastases receiving the bisphosphonate zoledronic acid. J. Clin. Oncol. 2005, 23, 4925-4935.

25. Ibrahim, T.; Sacanna, E.; Gaudio, M.; Mercatali, L.; Scarpi, E.; Zoli, W.; Serra, P.; Ricci, R.; Serra, L.; Kang, Y.; Amadori, D. Role of RANK, RANK-L, OPG and CXCR4 tissue markers in predicting bone metastases in breast cancer patients. Clin. Breast Cancer 2011, 11, 369-375.

26. Sacanna, E.; Ibrahim, T.; Gaudio, M.; Mercatali, L.; Scarpi, E.; Zoli, W.; Serra, P.; Bravaccini, S.; Ricci, R.; Serra, L.; et al. The role of CXCR4 in the prediction of bone metastases from breast cancer: A pilot study. Oncology 2011, 80, 225-231.

27. Terpos, E.; Szydlo, R.; Apperley, J.F.; Hatjiharissi, E.; Politou, M.; Meletis, J.; Viniou, N.; Yataganas, X.; Goldman, J.M.; Rahemtulla, A. Soluble receptor activator of nuclear factor kappaB ligand-osteoprotegerin ratio predicts survival in multiple myeloma: Proposal for a novel prognostic index. Blood 2003, 102, 1064-1069.

28. Mercatali, L.; Ibrahim, T.; Sacanna, E.; Flamini, E.; Scarpi, E.; Calistri, D.; Ricci, M.; Serra, P.; Ricci, R.; Zoli, W.; et al. Bone metastases detection by circulating biomarkers: OPG and RANK-L. Int. J. Oncol. 2011, 39, 255-261.

(C) 2013 by the authors; licensee MDPI, Basel, Switzerland. This article is an open access article distributed under the terms and conditions of the Creative Commons Attribution license (http://creativecommons.org/licenses/by/3.0/). 